\title{
Women Have Greater Density of Neurons in Posterior Temporal Cortex
}

\author{
S. F. Witelson, ${ }^{1,2,3}$ I. I. Glezer, ${ }^{4}$ and D. L. Kigar ${ }^{1}$ \\ Departments of ${ }^{1}$ Psychiatry, ${ }^{2}$ Biomedical Sciences, and ${ }^{3}$ Psychology, McMaster University, Hamilton, Ontario L8N \\ 3Z5, Canada and ${ }^{4}$ Department of Cell Biology and Anatomical Sciences, CUNY Medical School, New York, New \\ York 10031
}

Cytoarchitectonic area $T A_{1}$ (von Economo) in the cortex of the planum temporale within the Sylvian fissure, which is auditory association cortex and documented to be part of the neural substrate of language functions, was studied quantitatively in the brain specimens of five women and four men (mean age of $\mathbf{5 0}$ year). All cases were documented to be medically and cognitively normal, and consistently right-handed. We investigated the possibility that the difference in brain size between men and women is reflected in differences in the numerical density of neurons in area $T A_{1}$, an area associated with morphologic and psychological sex differences. Neuron counts were made directly through cell differentiation under the microscope from Nissl-stained sections. Cortical depth, the number of neurons through the depth of cortex under $1 \mathrm{~mm}^{2}$ of cortical surface $\left(N_{c}\right)$, and the number of neurons per unit volume $\left(N_{v}\right)$ were obtained for the total cortex and for each of the six layers in each hemisphere. For total cortex in both hemispheres, depth and $\boldsymbol{N}_{c}$ were similar, but $\boldsymbol{N}_{v}$ was greater by $11 \%$ in women, with no overlap of scores between the sexes. The sex difference in $\boldsymbol{N}_{v}$ was attributable to layers II and IV; in contrast, $\boldsymbol{N}_{\boldsymbol{v}}$ did not differ between the sexes in layers III, V, and VI. This is the first report of such a sex difference in human cortex. The results suggest that the cortical functional unit has a different ratio of input and output components in men and women which could have implications for the sex differences in cognition and behavior. Due to the small sample size and the homogeneity of the cases studied, generalizability of the results requires replication by other studies.

In addition, cytoarchitectonic mapping indicated that area $T A_{1}$ also occurs in the vertical posterior wall of the Sylvian fissure, providing evidence that anatomical definition of the planum temporale should include the posterior vertical wall of the superior temporal gyrus.

\footnotetext{
Received June 13, 1994; revised Nov. 7, 1994; accepted Nov. 10, 1994

This work was supported by NIH Contract NS-62344 and Grant NS-18954 Medical Research Council (Canada) Grant MA-10610, and grants from the J. P. Bickell Foundation, Toronto, and The EJLB Foundation, Montreal to S.F.W.). We are grateful to Dr. M. Colonnier, formerly of Laval University, PQ., for help in establishing the methods and with some cytoarchitectonic analyses; to Dr. H. Lansdell, NIH, who instigated and nurtured this work; Dr. C. H. Goldsmith, McMaster University, for statistical advice; Dr. M. Giguere for technical assistance; and the Departments of Pathology in Hamilton which provided the brain specimens.

Correspondence should be addressed to Dr. Sandra F. Witelson, Department of Psychiatry, McMaster University, 1200 Main Street West, Hamilton, Ontario L8N 3Z5, Canada.

Copyright 1995 Society for Neuroscience $0270-6474 / 95 / 153418-11 \$ 05.00 / 0$
}

[Key words: cerebral cortex/anatomy and histology, auditory cortex, cell count, brain mapping, sex characteristics, neurons/cytology, organ weight, sexual differentiation, language region, cortical layers, planum temporale, brain bank]

It is well established that brain size is approximately $10 \%$ larger in men than women (e.g., Dekaban and Sadowsky, 1978), but the significance of this disparity is not known. The difference in brain size is likely manifested in a difference in cortical volume, which in turn must be reflected in some sex difference in cellular characteristics, to date undelineated. The total number of cortical neurons depends on the number produced during proliferation and which ultimately survive early cell death (Rakic, 1988). This number might be the same in men and women, which could result in greater numerical density of neurons in women than men and therefore in different neuropil characteristics. Alternatively, factors in sexual differentiation of the brain (McEwen, 1983) could influence neuronal proliferation and survival such that numerical density is similar between the sexes, but the total number of neurons would be less in women than men. The microscopic differences in either scenario could have functional implications. Both models are likely too simplistic. Different laminar and columnar structures of the cortex may be affected by multiple factors operating differently in the sexes.

Sex differences have been documented in specific parts of the brain, such as size of the interhemispheric commissures. Area of the midsagittal section of the posterior region of the body of the corpus callosum (the isthmus) (e.g., Witelson, 1989; Habib et al., 1991; Steinmetz et al., 1992), and of the anterior commissure (Allen and Gorski, 1991; Witelson and Kigar, 1993) was found to be absolutely as well as relatively larger in women than men. In contrast, size of the total corpus callosum, the genu, and the splenium tends to be smaller in women (Witelson, 1989; Allen et al., 1991). In the primate brain, the interhemispheric axons that cross through the isthmus arise from and terminate in neurons in posterior temporal and parietal regions surrounding the posterior part of the Sylvian fissure (Pandya and Seltzer, 1986). These cortical regions also show sex differences in gross morphology in the human brain. The length of the horizontal segment of the posterior Sylvian fissure and the area of surrounding gyri such as the planum temporale (the superior surface of the posterior part of the superior temporal gyrus within the Sylvian fossa) are greater in both cerebral hemispheres in men than women, although length of other segments of the Sylvian fissure shows no sex difference (Witelson and Kigar, 1991, 1992; Aboitiz et al., 1992). 
Sex differences have also been found in the behavioral correlates of parietotemporal regions. Hand preference was found to be associated with size of the isthmus in men, but not in women (Denenberg et al., 1991; Habib et al., 1991; Witelson and Goldsmith, 1991), and with morphology of the posterior Sylvian fissure regions (Witelson and Kigar, 1992). Aphasia may follow left-sided lesions in this region less frequently in women than in men (Kimura, 1987). These results suggest that the functions mediated by parietotemporal regions may not be identical in men and women. Sex differences exist in cognitive functions such as verbal fluency and visuospatial perception (Halpern, 1992) which are mediated by these cortical regions (Hécaen and Albert, 1978). Such findings would be compatible with sex differences in the microscopic structure of parietotemporal cortex. However, little attention has been paid to the sex of the person in studies of the microscopic structure of human cortex. In an influential article, Rockel et al. (1980) concluded that basic uniformity exists in cortex in that the number of neurons in a column through the depth of the cortex is constant across mammalian species, within a species, and even among different cytoarchitectonic areas within the human brain except for striate cortex. However, they studied only two human brains, both male.

'I'his study investigated whether there are differences between men and women in quantitative aspects of the microscopic structure of postcrior temporal cortex. We focused our analysis on cytoarchitectonic area $\mathrm{T} A_{1}$ (von Economo and Koskinas, 1925) which covers the largest surface area of the posterior region of the superior temporal gyrus including the planum temporale, and which has a high degree of individual variability in its expanse (von Economo and Horn, 1930; Galaburda et al., 1978).

The specific aim of the study was to assess cortical depth and the numerical density of neurons for the full depth of the cortex and for each lamina in this cytoarchitectonic region in male versus female brain specimens. Additionally, since the posterior boundary of area $T A_{1}$ and of the planum temporale are not well documented, this study provided the opportunity to map the cytoarchitecture of parts of the superior temporal gyrus not studied previously.

\section{Materials and Methods}

\section{Brain specimen source}

The specimens were selected from a brain collection started in 1977 , designed for study of the relationship between structure and function in cognitively normal adults. The brains were obtained through autopsy from people with metastatic cancer who were essentially free of adverse signs of the disease when recruited as research subjects and who agreed to participate in a study involving both neuropsychological testing and, in the event of death, an autopsy to allow study of their brain. All subjects were documented to be within the limits of normal variation in cognitive function at the time of testing on the basis of detailed medical and social histories, medical consultations, and neuropsychological test results. Details of the recruitment procedure, neuropsychological testing, and characteristics of the brain collection are described elsewhere (Witelson and McCulloch, 1991).

The entire brain including the medulla was removed by the pathologist during autopsy following a standard procedure. Details are given elsewhere (Witelson and Kigar, 1992). Autopsies were done quickly, frequently within $2-3 \mathrm{hr}$ after death. After removal, the brain was suspended by the basilar artery and fixed in a $10 \%$ buffered formalin phosphate solution. Brain weight was measured at autopsy and again 3 weeks after fixation with a Sartorius balance (model U 3600).

\section{Selection of brain specimen sample}

Histologic analysis was done on a sample of nine brains (five women and four men), selected from a total of the 71 brain specimens available at the start of this study. Initially, 10 brains were selected, but one male brain had to be excluded because the required sections in one hemisphere proved to be oblique to the pial surface. Mean time interval between death and brain fixation at autopsy was $7.2 \mathrm{hr}$ for the women and $3.5 \mathrm{hr}$ for the men. All subjects were Caucasian. Aside from sex, an attempt was made to have as homogeneous a sample of brain specimens as possible, and cases were chosen to have the following criteria.

(1) All specimens were chosen from people who remained free of neurologic complications or any neuropsychiatric disorders until death.

(2) There was no gross or microscopic pathology observed on clinical neuropathologic examination.

(3) All specimens had a fixed brain weight that was no more than 1 SD below the mean value typical for that sex and chronologic age based on large groups (Dekaban and Sadowsky, 1978).

(4) All specimens were chosen from people who demonstrated on testing consistent-right-hand $(\mathrm{CRH})$ preference, defined as only righthand preference on a series of 12 items taken from the Annett (1967) handedness questionnaire. Subjects who showed any left-hand preference, even if they used their right hand for writing [classified as not consistent-right-handed (nonCRH)] were not included in this sample. The factor of hand preference was controlled because previous findings showed that the gross morphology of parietotemporal structures was different between CRH and nonCRH men (e.g., Witelson, 1989; Witelson and Kigar, 1992).

(5) Specimens were selected to have the more prevalent morphologic pattern of greater expanse of the planum temporale (PT) in the left than right hemisphere (e.g., Geschwind and Levitsky, 1968; Witelson and Kigar, 1988). Details of the definition of PT boundaries are given in the next section.

(6) Cases were selected from those who had extensive neuropsychological testing to enable future study of the relationships between microscopic structure and cognitive functions.

(7) Among the cases meeting the previous criteria, age at death was selected to make the two groups as comparable as possible.

Table 1 gives descriptive data for age, brain weight, and intelligence test scores for each sex group. The stringent criteria we used to select a sample for study has advantages and disadvantages. Although sample size is small, it was anticipated that the homogeneity among the cases might provide sufficient statistical power to detect even small differences that might exist. However, the specificity of the group in anatomical and psychological characteristics limits the generalizability of the results to the general population without additional studies.

\section{Location of cytoarchitectonic area $T \mathrm{~A}_{l}$; definition of planum temporale $(P T)$}

The tissue blocks for histologic study included cytoarchitectonic area $\mathrm{TA} A_{1}$ (von Economo and Koskinas, 1925), also referred to as area 22 (Brodmann, 1909; Blinkov, 1949) and area Tpt (Galaburda and Sanides, 1980). This area is found on the lateral surface of the posterior region of the superior temporal gyrus according to the schematic maps in these works and also on the posteriormost region of the superior surface of the supratemporal plane based on series of cytoarchitectonic maps of individual brain specimens (von Economo and Horn, 1930; Blinkov, 1949; Galaburda et al., 1978). The available maps clearly show that no sulcal boundaries exist to demarcate the limits of area $\mathrm{T} A_{1}$ in a particular brain a priori. Figure 1 presents a schematic map of the location of area $\mathrm{T} A_{1}$. This representation is a composite of previous maps with a modification based on our data (see Results), showing an extension of area $\mathrm{T} A_{1}$ onto the vertical wall of the posterior Sylvian fissure.

Area $\mathrm{T} A$, was studied in the superior surface of the posterior part of the superior temporal gyrus, that is, in the planum temporale (PT). The posterior boundary of PT has not been definitively established and is particularly ambiguous in some hemispheres. The Sylvian fissure bifurcates in most hemispheres (Witelson and Kigar, 1992) at point B into the posterior ascending and posterior descending rami. On the basis of previous work (Witelson, 1987; Witelson and Kigar, 1992), the posterior border of PT was defined as point $\mathrm{S}$, the end of the ascending ramus. The bifurcation divides PT into horizontal (HPT) and vertical (VPT) components (see Fig. 1). PT has been variously defined: ending at point B (e.g., von Economo and Horn, 1930; Geschwind and Levitsky, 1968), at point $\mathrm{D}$, the end of the descending ramus (Witelson and Pallie, 1973; Steinmetz et al., 1991) or at point $\mathrm{S}$ as in this study (also $\Lambda$ boitiz et al., 1992). In hemispheres in which the posterior upward swing of SF is very sharp and anterior in origin (see Witelson and Kigar, 1992, their 
Table 1. Descriptive data for the male and female groups of brain specimens

\begin{tabular}{|c|c|c|c|c|c|c|}
\hline & \multicolumn{3}{|c|}{$\operatorname{Men}(n=4)$} & \multicolumn{3}{|c|}{ Women $(n=5)$} \\
\hline & $\overline{\bar{X}}$ & (SD) & Min-Max & $\ddot{X}$ & (SD) & Min-Max \\
\hline Agc (ycars) & $48.8^{\prime \prime}$ & $(16.5)$ & $25-63$ & 53.6 & $(5.8)$ & $44-59$ \\
\hline Brain weight ${ }^{b}$ & 1425.1 & $(47.6)$ & $1384-1494$ & 1246.0 & $(45.7)$ & $1200-1292$ \\
\hline $\mathrm{FS} \mathrm{IQ}^{d}$ & 112.3 & (14.4) & $100-128$ & 110.6 & (2.7) & $108-115$ \\
\hline V IQ' & 110.0 & $(12.3)$ & $97-121$ & 111.2 & (7.5) & $103-123$ \\
\hline P IQ & 114.0 & $(17.0)$ & $97-135$ & 108.6 & $(11.0)$ & $93-122$ \\
\hline
\end{tabular}

"Comparison of sex groups: $t=0.65, p=0.54$.

${ }^{b}$ Whole brain weight at 3 week fixation in formalin. These values are similar to those for groups of comparable age in other studies (e.g., $1410 \mathrm{gm}$ for men and $1280 \mathrm{gm}$ for women; Dekaban and Sadowsky, 1978).

Comparison of sex groups: $t=5.74, p<0.001$.

"Full Scale, verbal, and performance IQ scores based on the Wechsler Adult Intelligence Scale (Wechsler, 1955) (comparison of sex groups: $p>0.50$ in each case).

Fig. $6 C$ ), PT has often been considered to be absent (e.g., Wada et al., 1975, their Fig. 1).

\section{Brain dissection and block removal}

In each hemisphere, the full extent of the Sylvian fossa was exposed by removing the frontal lobe en bloc and then cutting from $S$, the end of the SF (see Fig. 1), through the parietal and occipital lobes to remove the postcentral part of the parietal lobe including the parietal operculum. This dissection exposed Heschl's transverse gyrus and the full PT in the supratemporal plane and in the upward curve of SF. In this sample of nine brains, $56 \%$ of hemispheres had both HPT and VPT components. Three hemispheres (two male, one female) had only a VPT segment (no HPT) because point $B$, the point of bifurcation of SF, was

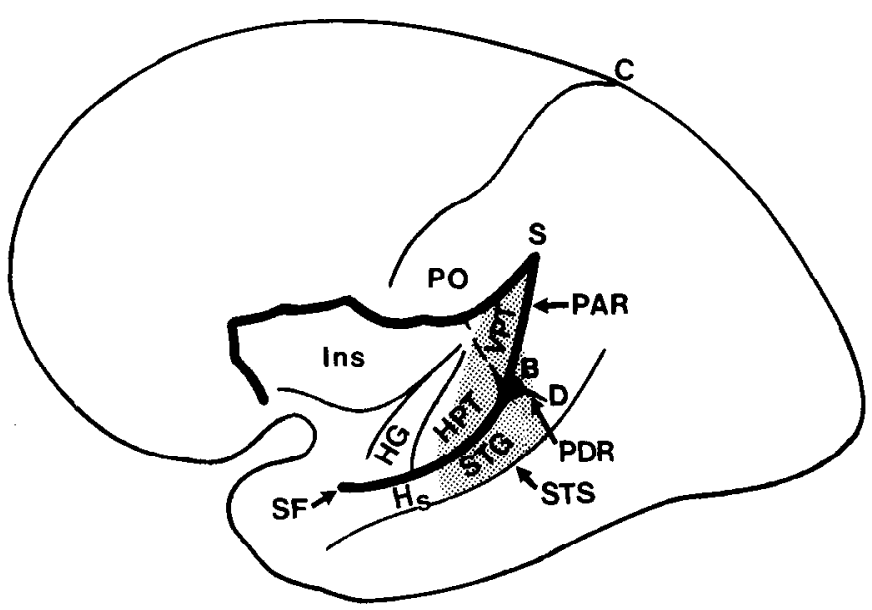

Figure 1. Schematic representation of the gross morphologic features of the planum temporale $(P T)$ and its surround and of the location of cytoarchitectonic area $\mathrm{T} A$, based on von Economo and Koskinas (1925) and on our modification. A left hemisphere with typical morphology is shown, with the upper and lower walls of the Sylvian fossa pulled apart (heavy lines represent the lateral edges), exposing the superior surface of the superior temporal gyrus. The Sylvian fissure $(S F)$ bifurcates at point $B$, into the posterior descending ramus $(P D R)$ ending at $D$, and the posterior ascending ramus $(P A R)$ ending at $S$. The floor of the Sylvian fossa exposes Heschl's gyrus $(H G)$ and the two components of the full PT (divided by the dashed line). The horizontal segment (HPT) is in the supratemporal plane, typically considered to be PT, and the vertical segment of the planum temporale $(V P T)$ is on the inner (posterior) wall of the upward curve of PAR. $C$, Dorsal end of the central sulcus; $H$, end of Heschl's transverse sulcus on the lateral aspect of the brain; Ins, insula; $P O$, parietal operculum; $S T G$, superior temporal gyrus; STS, superior temporal sulcus. The localization of area $\mathrm{T} A_{1}$ is represented by the stippled area. Our modification is the inclusion of $\mathrm{T} A_{1}$ in the cortical wall in VPT. congruent with Heschl's sulcus, an anatomic variant described elsewhere (Witelson and Kigar, 1992, their Fig. 6C).

A large block of the superior temporal gyrus was removed from each hemisphere. The block included the full superior surface of Heschl's gyrus and the full PT extending all the way to $\mathrm{S}$, thus including both HPT and VPT (Heschl/PT block). The block was bounded inferiorly by the superior temporal sulcus (see Fig. 2). If SF curved sharply at point $B$, it was sometimes necessary to cut the Heschl/PT block into two parts at point $\mathrm{B}$ and section them separately. The sample of area $\mathrm{TA}$ used for quantitative measurement was taken in most cases from HPT since it tended to have a greater expanse of area $\mathrm{T} A$, with a flat surface suitable for cell counts in sections cut perpendicular to the pia.

Photographs of the Heschl/PT block were taken to measure surface areas for use in obtaining estimates of the surface area of $\mathrm{T} A_{1}$ and subsequently estimates of cortical volume and the total number of neurons in area $\mathrm{T} A_{1}$. For each hemisphere, three photographs were taken, of HPT, VPT, and the lateral aspect of the block. HPT and VPT were photographed separately since the planes of the two segments are almost always different. The lateral area of $\mathrm{T} A_{1}$ was defined as the region between the extensions of Heschl's sulcus and of point B to the superior temporal sulcus (see Fig. 1). $\Lambda$ Il photographs were made at $1 \times$ magnification based on two orthogonal rulers in the photograph. Area measurements were made using the Bioquant II Digitizing Morphometry Program A5-IA2.

The sum of these three areas was used as the surface area of $\mathrm{T} A_{1}$. Since the boundaries of area $\mathrm{T} A_{1}$ do not necessarily coincide with sulci, this definition of the extent of $\mathrm{T} A_{1}$ is a very rough approximation. The estimate necessarily includes part of cytoarchitectonic area TB in HPT; the estimate does not capture the undulations of the surface of PT, nor the cortical surface within small sulci, such as the posterior descending sulcus (see Figs. 1,2), and the lateral measure uses gross anatomical features to define an unknown extent of the cytoarchilectonic area.

\section{Histological sectioning and staining}

Since morphology varies among hemispheres, the plane of cut for each block was chosen individually in an attempt to have sections made through a flat posterior PT region and angled perpendicularly to the pia in that region. Generally, the Heschl/PT block was sectioned in a plane perpendicular to the longitudinal axis of Heschl's transverse sulcus (see Fig. 2).

The whole block was frozen with dry ice and cut on a sliding microtome (American Optical) at a constant setting to yield final stained sections with a mean thickness of $25 \mu \mathrm{m}$. Pilot work had revealed that sections this thick were needed for recognition of cyloarchitectonic patterns. Every consecutive tenth section was saved and mounted. The orientation of each section in respect to its position in the brain was recorded. The sections were Nissl-stained with $0.1 \%$ cresyl violet solution. These sections were used to identify area $\mathrm{T} A_{1}$ and for quantitative analysis. Mean linear shrinkage of the sections through the depth of the cortex was $8.1 \%$, based on assessment of seven tissue blocks from four brains. 


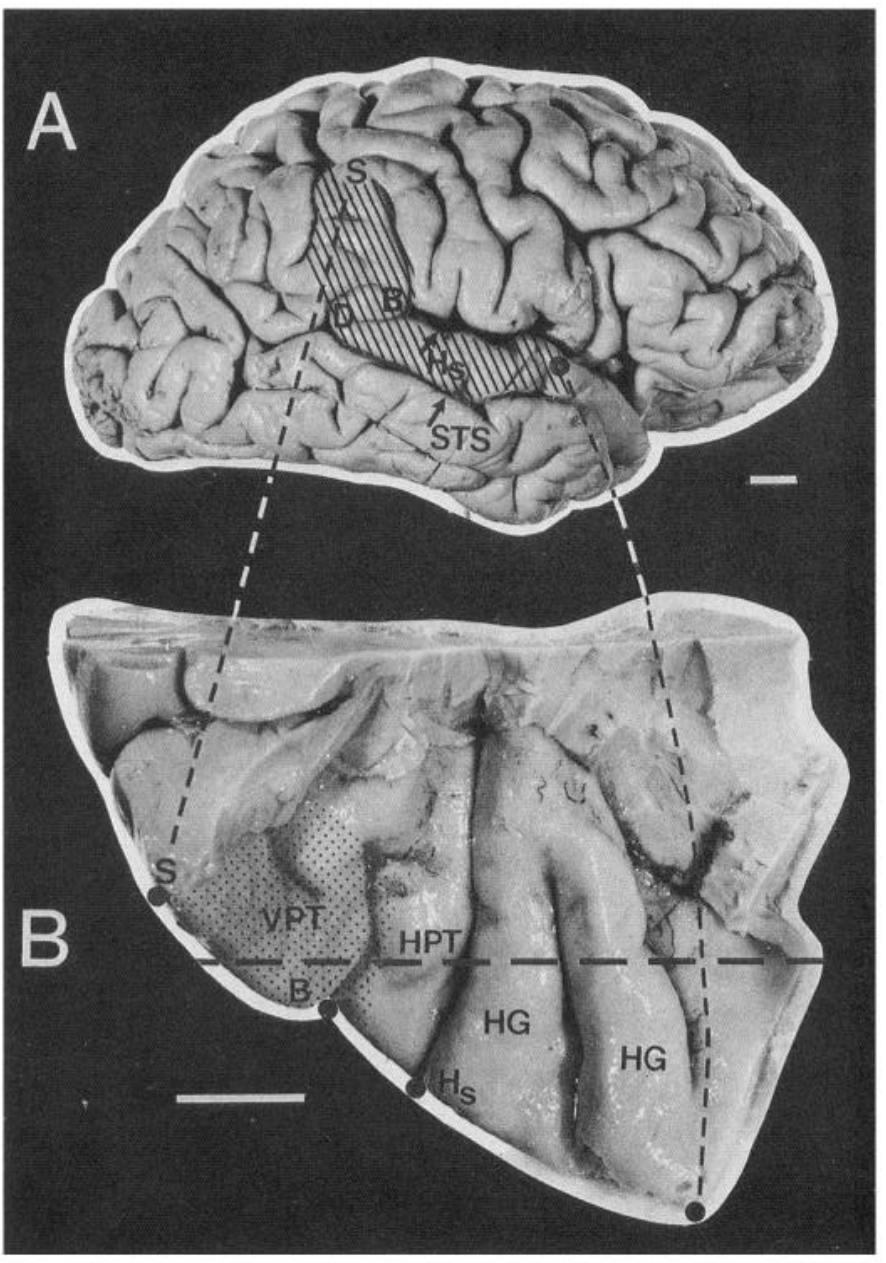

Figure 2. Photographs of the lateral view of a right hemisphere $(A)$ and the superior view $(B)$ of the Heschl/planum temporale $(H P T)$ block removed from that hemisphere. In this hemisphere, the horizontal and vertical components of PT could be removed in the same block. This photograph is in the plane of HPT; VPT is foreshortened. The brain was cut through PAR to reveal VPT. Point $B$ indicates where PDR meets the lateral edge. It can be seen that PDR does not extend as far medially as does PAR. The dashed lines connect anterior and posterior points in the H/PT block to their position in the hemisphere. The lateral aspect of the whole block is indicated by hatched lines in $A$. The plane of cut of the Nissl-stained sections (heavy dashed line) is indicated. The general location of cytoarchitectonic area T $A_{1}$ in PT is indicated by the stippled region. Abbreviations are as in Figure 1. Scale bars, $1 \mathrm{~cm}$.

\section{Cytoarchitectonic analysis}

To ensure our ability to distinguish among the different cytoarchitectonic areas in the region of PT, and specifically to identify area $\mathrm{T} A_{1}$ as described by von Economo and Koskinas (1925), we studied all the serial sections of Heschl/PT blocks, including the HPT and VPT components, prepared as described in previous sections, for three brains (six hemispheres). This included approximately $80-100$ sections per hemisphere and cytoarchitectonic mapping was done for the full extent of each slide.

We were able to distinguish four main cytoarchitectonic regions comparable to von Economo's areas $\mathrm{T} C, \mathrm{~T} B C, \mathrm{~T} B$, and T $A_{1}$. They occurred always in the same topographic progression in a general anterior to posterior direction over the Heschl and PT gyri. Figure 3 presents a photograph of a typical section of a Heschl/PT block showing the location of the four major cytoarchitectonic areas. We found, as shown in von Economo and Horn's (1930) detailed maps, that area TC, primary koniocortex, which occurs in islands of tissue, and area $\mathrm{T} B C$, a transitional form, are typically located within Heschl's gyrus; area T $B$ covers the anterior portion of HPT; and area $\mathrm{TA}_{1}$ is located posterior to area TB in HPT and also in VPT when present.

Differentiation of these areas was based on visual qualitative cytoarchitectonic characteristics such as laminar distribution of cells, cell size, packing density, columnar arrangement, and relative thickness of different layers. Using these criteria, area $\mathrm{T} A$, was identified in sections from each hemisphere of the nine brains studied. Identification was done for each brain independently by three raters. In each case, there was $100 \%$ agreement as to which cortical region was area $\mathrm{T} A_{1}$.

\section{Quantitative analyses}

The quantitative analyses used in this study were based on a computerassisted manual method involving cell differentiation in Nissl-stained sections under the microscope, marking neurons having nucleoli with the aid of a camera lucida, and defining laminar depths using photomicrography. The strict criterion of counting only neurons with nucleol and the use of thick sections in which most neurons could be seen in three dimensions to aid differentiation of small neurons versus glial cells help obtain accurate estimates of neuron counts. This direct method, although time consuming, was chosen rather than automatic methods based on computer detection of items according to size (e.g., Terry et al., 1987) because the latter methods likely have more measurement error which could obscure the size of the individual differences that might exist and were being investigated.

The cortical sites of area T $A_{1}$ used for measurement were selected so that each site was not at the crest or valley of a gyrus, the pial surface was flat, the cell columns were perpendicular to the cortical surface, and the least amount of technical artifacts and blood vessels were present. Six slides per hemisphere were selected for measurement. In each slide, area $\mathrm{T} A_{1}$ was identified by three raters, and two adjacent traverses, each $125 \mu \mathrm{m}$ wide, were analyzed. The sample size of 12 traverses per hemisphere was selected because pilot work had indicated that the variation in cell counts within a hemisphere was not reduced with a number of traverses greater than twelve.

Cortical and laminar depth. Low power photomicrographs of the sites chosen for measurement for each slide were used to delineate the six cortical layers. The common cytoarchitectonic features such as packing density of the cells and their size and arrangement in cytoarchitectonic columns were used for defining the boundaries between the cortical layers. The boundary between the cortex and subcortical white matter was defined by the presence of no more than three neurons in the last counting field. For each photomicrograph, there was a paired photograph of the stage micrometer taken at the same magnification, which was used as a ruler to measure the depth of each layer. Using the magnification factor, laminar divisions were indicated on the camera lucida drawings to calculate the number of neurons in each lamina.

Neuronal counts. Neurons were counted in two adjacent traverses from the pia to the edge of the white matter. Neurons were differentiated from glia under high magnification $(630 \times)$, using typical features such as general size of the cell, size and structure of the nucleus, and distribution of the nuclear chromatin. Those neurons having nucleoli were counted by marking them via a camera lucida onto sheets, each sheet representing one microscopic field. Errors in counting (double counting or omission) were kept in check with the aid of an eyepiece grid (10 $\mathrm{mm}$ by $10 \mathrm{~mm}$ ) that was used to outline consecutive microscopic fields through the depth of the cortex. The frame of the grid defined the microscopic field of 125 by $125 \mu \mathrm{m}$ on the slide as seen at the magnification used. The neurons within the grid and those which crossed the top and right-side borders of the frame were counted. The region of cell counting could be found again by marks made directly on the slide and by tracings of artifacts made on the camera lucida drawings. This allowed ambiguous cells to be checked with a second investigator. The number of neurons was counted for each layer from the tracings with the aid of a computer. All cell counts were corrected using an Abercrombie correction to adjust for the varying thickness among sections. Intrajudge reliability revealed counts within $1-2 \%$ for the same traverse done on different days. Interjudge reliability was checked in a few cases and was found to be within $2-5 \%$.

Section thickness. Numerical densities were calculated from the raw count numbers for each slide by using the measured section thickness for that slide. Although the frozen tissue was cut at a constant setting, section thickness varied among the slides. Thickness was measured at six different sites through the depth of cortex for each slide. This was done under oil immersion by focusing from the top to bottom of the section and reading the values from the fine focus knob of the micro- 


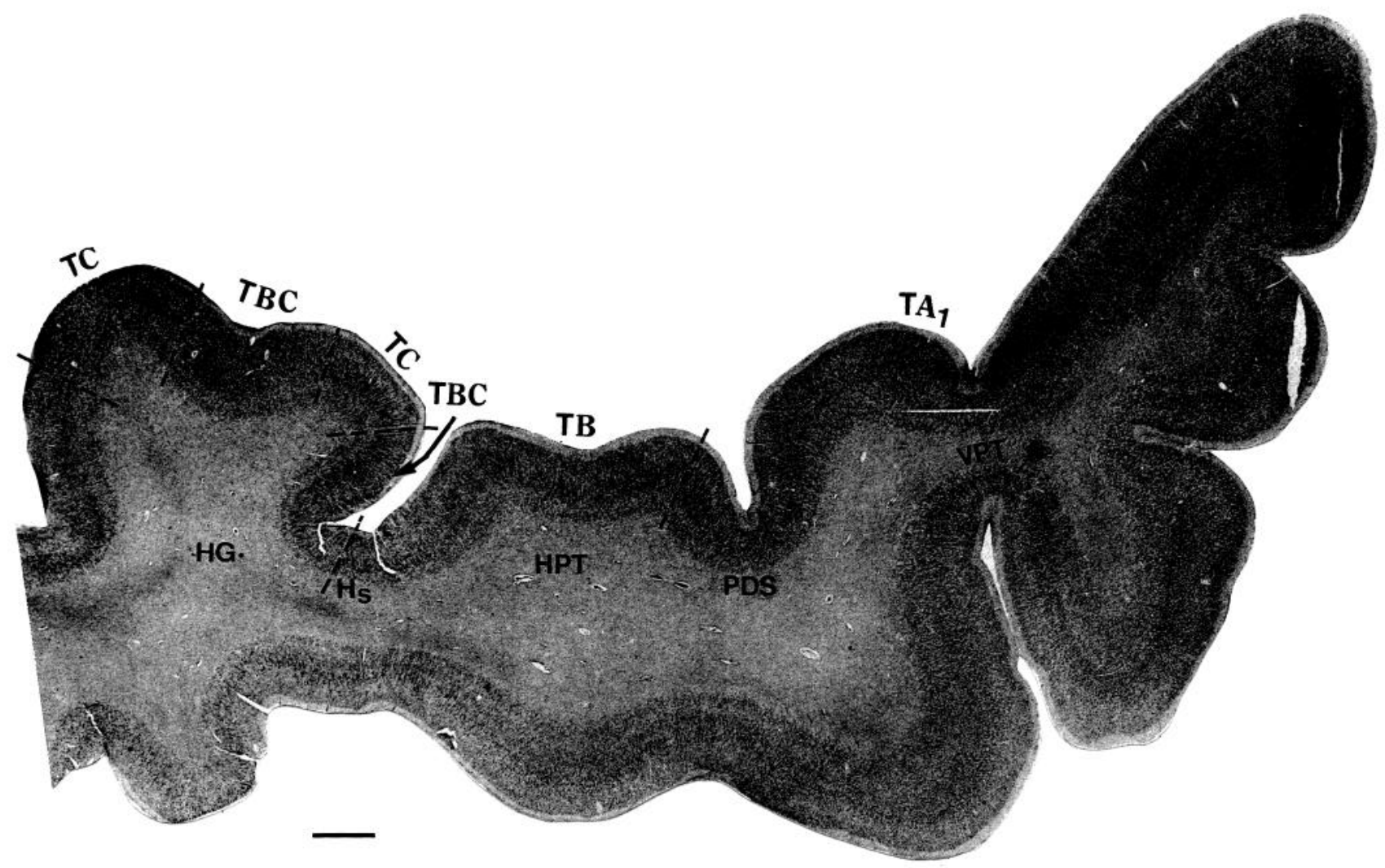

Figure 3. Photograph of a Nissl-stained section through a Heschl/planum temporale (HPT) block showing the location of the four major cytoarchitectonic regions, labeled as in von Economo and Koskinas (1925). The section is from the H/PT block shown in Figure 2 . TC is primary auditory koniocortex and occurs in islands; TBC is a transitional form, both found mainly in Heschl's gyrus $(H G)$; T $B$ is association cortex which covers the anterior region of the PT posterior to Heschl's sulcus $\left(H_{s}\right) ; \mathrm{T} A_{1}$ is association cortex found in the posterior regions of PT (in HPT and VPT). See Results for a description of the cytoarchitectonic characteristics of area $\mathrm{T} A_{1}$. Abbreviations are as in Figure 1. Scale bar, $2 \mathrm{~mm}$.

scope. Section thickness was calculated based on the focus knob reading and the refractive index of oil (1.515). For each slide, the mean value of the six measures was obtained. Mean section thickness for all slides was $24.9 \mu \mathrm{m}(\mathrm{min} / \max =17.0 / 34.6)$.

Histologic variables. The following measures were obtained for each slide.

(1) Cortical depth, depth throughout the cortex (total depth) and depth of each cortical lamina (micrometers): this variable was obtained by direct measurement and for each slide is the mean value for the two traverses.

(2) $N_{c}$, number of neurons under $1 \mathrm{~mm}^{2}$ of cortical surface through the total depth of the cortex, and through the depth of each layer: this is columnar neuronal number, that is, an estimate of the number of cells in a column perpendicular to the pial surface with volume equal to I $\mathrm{mm}^{2} \times d \mathrm{~mm}$, where $d$ is either total cortical depth or laminar depth.

This variable was calculated for each slide according to the equation $N=n /(w * t)$, where $N_{c}$ is the number of neurons under $1 \mathrm{~mm}^{2}$ of cortical surface, $n$ is the mean number of neurons from two traverses, $w$ is the width of the traverse (in $\mathrm{mm}$ ), and $t$ is the mean section thickness (in $\mathrm{mm}$ ).

(3) $N_{1}$, number of neurons per unit volume $\left(1 \mathrm{~mm}^{3}\right)$ for total cortical depth and per unit volume for each layer (often called cell packing density): for total cortical depth, $N_{v}$ is the value for the total column, not the mean of the six laminar values for $N$.

This variable was calculated for each slide according to the equation $N=n /(d * w * t)$, where $N$ is the number of neurons per $1 \mathrm{~mm}^{3}$ of cortical tissue, $n$ is the mean number of neurons from two traverses, $d$ is the mean cortical or laminar depth of the two traverses (in $\mathrm{mm}$ ), $w$ is the width of the traverse (in mm), and $t$ is the mean section thickness (in $\mathrm{mm}$ ). Abercrombie corrections were applied here.

For each slide, the depth and numerical density values, in combination with the estimates of $\mathrm{T} A_{1}$ surface area, were used to calculate estimates of the cortical volume of area $\mathrm{T} A_{1}$ and the total number of neurons in $\mathrm{T} A_{1}$ and in each layer of $\mathrm{T} A_{1}$. For each hemisphere, the value for each variable was the mean of the scores from the six slides. The final score used in the statistical analyses was the mean of the two hemisphere scores (see next section).

The mean value for $N$ obtained for the sample of four male brains in this study is almost identical to that reported by Rockel et al. (1980) for two brains using a similar method (see Witelson et al., 1992). The mean values obtained for $N_{\text {r }}$ were well within the range of $N_{\text {r }}$ values reported for various cortical regions in recent studies using the optical dissector method (Braendgaard et al., 1990; Pakkenberg, 1993). [A few older studies using manual methods reported $N_{r}$ values, but often only on single cases, with sex unspecified, or extent of tissue shrinkage not indicated (see Blinkov and Glezer, 1968).] These comparisons provide some external validation for the methods and representativeness of the sample in the present study.

\section{Statistical procedures}

Descriptive statistics were obtained for all variables. Two-way analyses of variance (ANOVA) were done for each variable with Sex and Hemisphere as between factors. There was no interaction between factors; in other words, the sex differences were the same for each hemisphere. This report addresses the factor of Sex. Accordingly, all scores represent the mean of the scores for the two hemispheres of each brain. The factor of Hemisphere itself will be the subject of a subsequent report. All percent difference scores were calculated using the mean of the two scores for the denominator $([(F-M) /[(F+M) / 2]] \times 100)$, where $F$ $=$ female and $M=$ male. Independent two-tailed Student's $t$ tests and Pearson product-moment correlations were used with significance level set at $\alpha=0.05$. Differences of at least $10 \%$ were considered biologically important and were reported as a tendency even if they were not statistically significant with the present small sample size. 


\section{Results}

\section{Cytoarchitectonic analysis}

Area $\mathrm{T} A_{1}$ was found in the posterior region of PT in each hemisphere and corresponded to the pictorial and verbal description of other authors (e.g., von Economo and Koskinas, 1925). As reported in previous studies (von Economo and Horn, 1930; Galaburda et al., 1978), there was much individual variation in the location and extent of area $T A_{1}$. Cortical tissue was analyzed in the horizontal supratemporal surface (HPT) and in the surface of the posterior wall of the Sylvian fossa when it curves upwards within the posterior ascending branch of SF (VPT) (see Fig. 1). It was found, as documented previously, that area $T A_{1}$ is located in the posteriormost region of HPT. TA $A_{1}$ cortex was found also in VPT (see Fig. 1). Of particular relevance for cytoarchitectonic mapping, $\mathrm{T} A_{1}$ tissue was evident in VPT cortex in each of the three hemispheres in which there was no HPT.

Area $T A_{1}$ can be recognized by the following characteristic cytoarchitectonic features. Radial striation is well expressed and in most cases radiation extends from the upper part of layer III into layer VI. However, these cytoarchitectonic columns are less regular than in area $\mathrm{T} B$, and the radial striation in layers $\mathrm{V}$ and VI is less evident than in area $\mathrm{T} B$. The border between layers II and III is much less distinct than in area TB. Layer III is composed of wide columns (about 3-4 cells across) of pyramidal cells of a progressively larger size (50 $\mu \mathrm{m}$ or more) until the border at which there is intermingling with granular cells of layer IV. The cells in layer V are larger than in layer VI, but their density is less than in layer VI and than in layer $V$ of area $\mathrm{T} B$. Figure 4 presents photomicrographs of a sample of area $\mathrm{T} A_{\text {, }}$ from one female and one male brain. No sex differences were evident by qualitative observation.

Table 2 presents means, SDs, and statistical test results for the three variables (depth, $N_{c}, N_{v}$ ) for the total cortex and for each layer for each sex group.

\section{Cortical depth}

As shown in Table 2, cortical depth did not differ between the sexes for either total cortex or for any layer. Figure $5 \mathrm{~A}$ shows the percent difference between the sexes for cortical depth. There was a tendency for layer III to be greater in men than women by $11 \%$, and a suggestion of a similar tendency for layers I, V, and VI. In contrast, layers II and IV showed virtually no difference between the sexes.

\section{$\mathrm{N}_{\mathrm{c}}$, number of neurons under $1 \mathrm{~mm}^{2}$ of surface through the depth of cortex (columnar neuronal number)}

As shown in Table 2, $N_{c}$ did not differ statistically between men and women for total cortex or for any layer. Figure $5 B$ shows the percent difference between the sexes. $N_{c}$ tended to be greater in women by approximately $12 \%$ in layer II and by $17 \%$ in layer IV. $N_{c}$ for the other layers was very similar in both sexes.

\section{$\mathrm{N}_{\mathrm{v}}$, number of neurons per unit volume (cell packing density)}

As shown in Table 2, $N_{v}$ was greater in women than men for the total cortex $(p=0.008)$ and for layer IV $(p=0.02)$. There was a tendency for layer $I 1$ to be greater in women $(p=0.08)$. Figure $5 C$ shows the percent difference between the sexes for each layer. $N_{v}$ was grcater in women by $11 \%$ for the total cortex, by $13 \%$ in layer II and by $20 \%$ for layer IV.

The quantitative analyses revealed a similarity in values for $N_{v}$, between layers II and IV, and between layers III and V (see Table 2). This was so in each sex. $N_{v}$ for the total cortex did not show an association with chronological age in our samples $(r=$ -0.15 for women, $r=-0.05$ for men).

Figure 6 presents the plots of the values of $N_{\text {. }}$ for each brain for the total cortex, for layer II and for layer IV. There was no overlap between the sexes in $N_{v}$ for total cortex (Fig. 6A). In the case of layers II and IV, only one male brain had a value within the interval observed for the female brains (Fig. 6B,C). One man was considerably younger ( 25 years) than all other cases. He was one of the middle data points in the male group in Figure $6, A$ and $B$, and the highest value in Figure $6 C$.

\section{Granular versus nongranular layers}

The sex differences in $N_{v}$ occurred in layers II and IV (referred to as the granular system), and not in layers III, V, and VI (referred to as the nongranular system). Based on this general pattern, we investigated the relationship between these two parts of the cortical plate (layer I was excluded from this analysis). The mean value of $N_{v}$ for layers II and IV was plotted against the mean value of $N_{v}$ for layers III, V, and VI for each brain (Fig. 7). There was a discrete separation between the sexes, as highlighted by the stippled region within the scatterplot. The graph shows the lack of overlap between the sexes for granular system $N_{1}$. represented on the y-axis, in contrast to the almost complete overlap of male and female scores for nongranular system $N_{\text {v }}$ represented on the $\mathrm{x}$-axis. The straight-line relationship between the granular and nongranular values for $N_{v}$ for men was $r=$

$-0.78(p=0.22)$, and for women was $r=-0.29(p=0.64)$. The stippled region within the scatterplot highlights the possibility of curvilinear relationships.

\section{Estimated total number of neurons in area $T A$,}

The total number of neurons in area $\mathrm{T} A_{1}$ was estimated by the formula $N_{T}=a * d * N_{v}$, where $N_{T}$ is the total number of neurons in area $\mathrm{T} A_{1}, a$ is the estimated total surface area of area $\mathrm{T} A_{1}$, defined as the sum of areas of the two components of PT (HPT, VPT) and of the lateral expanse of the posterior part of the superior temporal gyrus (as defined in Materials and Methods), $d$ is the total cortical depth, and $N_{v}$ is the numerical density of neurons per unit volume (as defined in Materials and Methods). The mean estimated surface area of $\mathrm{T} A$, was $1086 \mathrm{~mm}^{2}$ and 916 $\mathrm{mm}^{2}$ in men and women, respectively (17\% difference, $t=0.76$; $p=0.47$ ). The mean estimated cortical volume of $\mathrm{T} A_{1}$ was 3146 $\mathrm{mm}^{3}$ and $2513 \mathrm{~mm}^{3}$ for men and women, respectively (22\% difference, $t=1.02 ; p=0.34$ ).

Table 3 presents $N_{r}$ for the total cortex and for each layer for each sex. Percent sex differences are also given. No differences reached statistical significance, but $N_{T}$ in each of the nongranular layers (III, V, and VI) tended to be greater in men than women by about $12 \%$ or more. In each granular layer, $N_{T}$ was very similar between men and women. Table 3 also gives $N_{T}$ per layer as a percent of $N_{T}$ for the total arca $\mathrm{TA}_{1}$ cortcx.

\section{Discussion}

Sex differences in cell packing density

Cell packing density through the full depth of the cortex in cytoarchitectonic area $\mathrm{T} A_{1}$ in both hemispheres was found to be $11 \%$ greater in the brains of five women compared to four men, all documented to be cognitively normal and consistently righthanded. There was no overlap in $N_{v}$ between the sexes (see Fig. $6 A$, Results). To our knowledge this is the first report of a sexual dimorphism in a quantitative microscopic feature of human cortex. These results provide a first step to delineating a cellular 

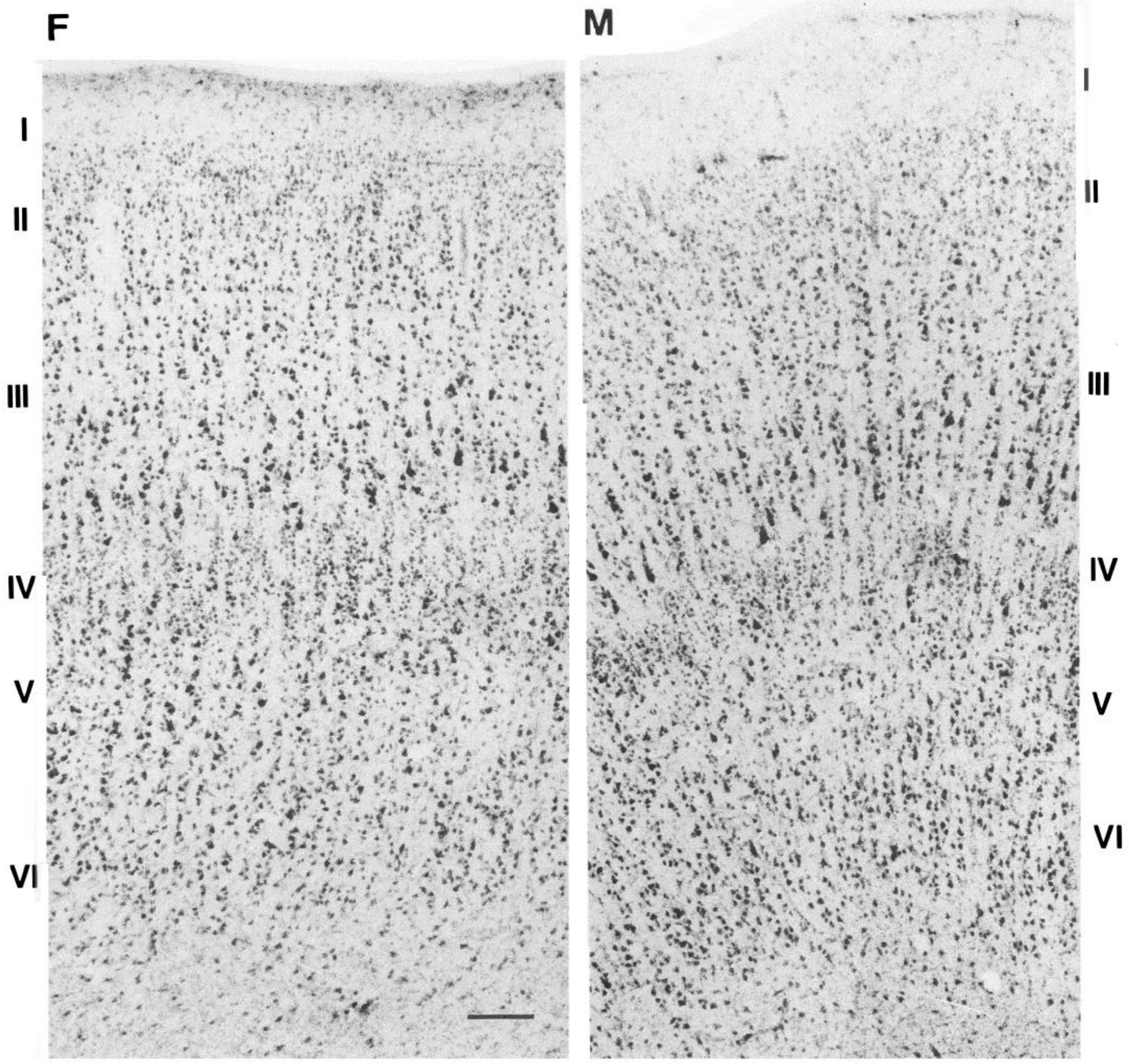

Figure 4. Photomicrographs of Nissl-stained sections showing cytoarchitectonic area $\mathrm{T} A_{1}$ (described in Results) in the posterior region of the planum temporale in the superior temporal gyrus in one female $(F)$ and one male $(M)$ brain. Each micrograph represents a section through the full depth of the cortex cut perpendicular to the pia and the six cortical layers are indicated. The width of the transverse for counting was $250 \mu \mathrm{m}$. No sex differences were evident by qualitative observation. Scale bar, $200 \mu \mathrm{m}$.

basis that might underlie the sex difference in overall brain size, a difference which is well documented but whose significance is not known. The sex difference in $N_{v}$ may reflect differences in connectivity and synaptology which could have physiological and behavioral consequences.

Neither total cortical depth nor the number of neurons in a column of cortex under $1 \mathrm{~mm}^{2}$ of cortical surface $\left(N_{c}\right)$ was different between men and women. The similarity in $N_{c}$ is consistent with the hypothesis of uniformity of cortex for $N_{c}$ (Rockel et al., 1980).

The results in this study appear clear, but they are based on a small sample and need replication in other samples to gain greater confidence. Additionally, because the sample studied was selected to be homogeneous in several specific characteristics, such as hand preference, caution must be taken about generalizing the results to the entire population before broader samples are investigated. In this regard, our preliminary results with a sample of six left-handers are relevant as they also indicate greater $N_{v}$ in women than men. It also remains to be determined whether the observed sex difference extends to cortex in other regions of the brain.

Some considerations support confidence in these findings. The 
Table 2. Means ${ }^{a}$ and SDs for cortical depth, $N_{c}$, and $N_{v}$ for the samples of five women and four men

\begin{tabular}{|c|c|c|c|c|c|c|}
\hline \multirow[b]{2}{*}{ Layers } & \multicolumn{2}{|c|}{ Cortical depth $(\mu \mathrm{m})$} & \multicolumn{2}{|c|}{$N$ (in thousands) } & \multicolumn{2}{|c|}{$N_{\mathrm{r}}$ (in thousands) } \\
\hline & $\begin{array}{l}\text { Women } \\
\bar{X}(\mathrm{SD})\end{array}$ & $\begin{array}{l}\text { Men } \\
X(\mathrm{SD})\end{array}$ & $\begin{array}{l}\text { Women } \\
X(\mathrm{SD})\end{array}$ & $\begin{array}{l}\text { Men } \\
X(S D)\end{array}$ & $\begin{array}{l}\text { Women } \\
\bar{X}(\mathrm{SD})\end{array}$ & $\begin{array}{l}\text { Men } \\
\bar{X}(\mathrm{SD})\end{array}$ \\
\hline I & $276(26)$ & $293(51)$ & $4(1)$ & $4(2)$ & $13(4)$ & $13(3)$ \\
\hline II & $275(30)$ & $277(19)$ & $25(3)$ & $22(4)$ & $91(2)$ & $81(12)^{*}$ \\
\hline III & $810(117)$ & $902(104)$ & $38(5)$ & $39(8)$ & $47(4)$ & $44(6)$ \\
\hline IV & $320(41)$ & $316(25)$ & $27(4)$ & $23(5)$ & $83(7)$ & $68(9)^{* *}$ \\
\hline $\mathrm{V}$ & $433(76)$ & $469(142)$ & $19(3)$ & $20(7)$ & $45(2)$ & $42(3)$ \\
\hline VI & $622(70)$ & $666(79)$ & $18(2)$ & $18(2)$ & $27(4)$ & $25(4)$ \\
\hline Total & $2735(156)$ & $2923(341)$ & $130(7)$ & $125(16)$ & $48(2)$ & $43(1)^{* * *}$ \\
\hline
\end{tabular}

$N_{\text {. is }}$ the number of neurons under $1 \mathrm{~mm}^{2}$ of cortical surface; $N_{\mathrm{v}}$ is the number of neurons per $\mathrm{mm}^{3}$ of tissue.

"Scores are the means of the right and left hemisphere values.

$*, p=0.08$

$* *, p=0.02$

$* * *, p=0.008$, for independent $t$ tests, $\mathrm{df}=7$.

variation of $N_{r}$ scores within each sex was small, as evidenced by the low SDs ( $2-4 \%$ of the mean scores), suggesting that the mean scores may be good estimates of the true numerical densities for each sex. The fact that the $N_{\mathrm{v}}$ difference occurred only in one type (granular) of layer suggests that the finding is not due to a methodological artefact. The mean $N_{v}$ values we obtained of 48,000 and 43,000 (see Table 2) for women and men, respectively, are quite comparable to $N_{v}$ values obtained with larger samples in studies using the optical dissector method. Bra-

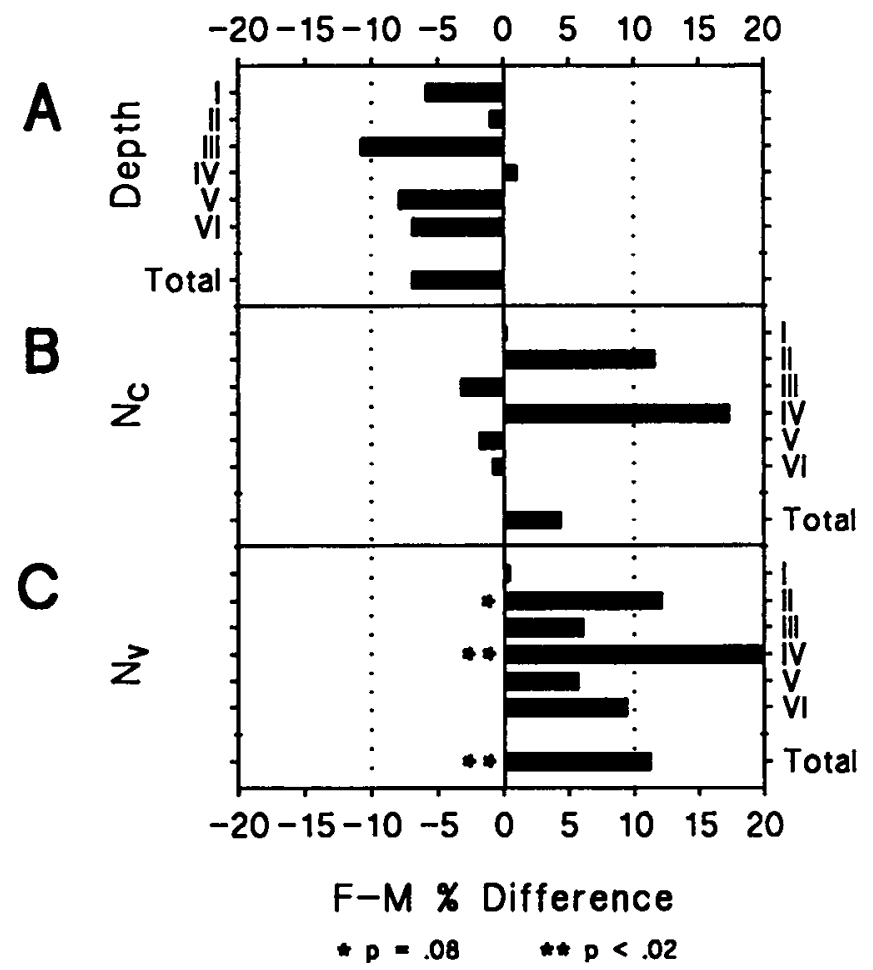

Figure 5. Bar graphs depicting the percent difference between female $(F)$ and male $(M)$ brains for cortical depth $(A), N_{c}(B)$, and $N_{v}(C)$ for total cortex and for each layer for area $T A_{1}$. Scores are means of right and left hemispheres. The percent difference is $[(F-M) /[(F+M) / 2]]$ $\times 100 . N_{c}$ is the number of neurons under $1 \mathrm{~mm}^{2}$ of cortical surface. $N_{v}$ is the number of neurons per $1 \mathrm{~mm}^{3}$ of tissue. The dotted lines highlight differences of at least $10 \%$. endgaard et al. (1990) calculated a mean value for $N_{v}$ for temporal cortex (cytoarchitectonic region not specified) of approximately 46,000 in a sample of five 80 year old male brains ( $\mathrm{min}$ / $\max =38,000 / 53,000)$. Pakkenberg (1993) reported a mean value of $N_{v}=40,400$ for temporal cortex for a group of 16 men having a mean age of 60 years. In a preliminary study involving 18 men and 8 women, Pakkenberg et al. (1989) reported only one mean value $\left(N_{\mathrm{v}}=45,000\right)$ for the overall cortex, including occipital cortex. They stated no variation was observed with age or sex but no values were reported.

While the results reported here must be verified in other studies before they can be generally accepted, the following comments are offered. The magnitude of $11 \%$ for the sex difference in $N_{\mathrm{r}}$ for the total cortex corresponds closely to the $10 \%$ sex difference in brain size (Dekaban and Sadowsky, 1978). Such a result raises the hypothesis that one possible cause of the greater $N_{r}$ in women is a simple mechanical compression or geometric consequence of a smaller brain. The sex difference in brain size

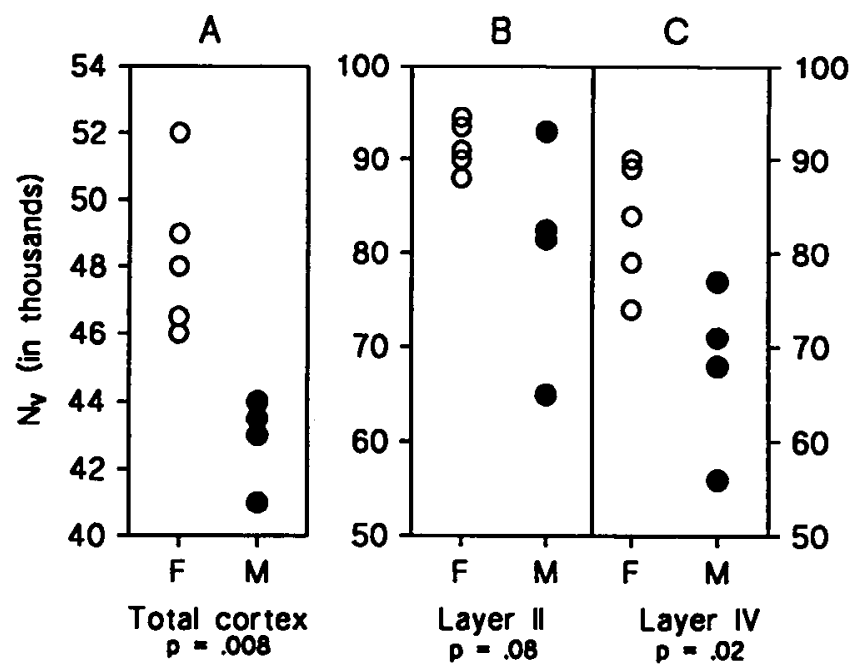

Figure 6. Plot of values for $N_{1}$ in area $\mathrm{T} A_{1}$ for each of the five female $(F)$ (open circles) and four male $(M)$ (solid circles) brains studied. Scores are means of right and left hemispheres. $A$, Total cortex; $B$, layer II; $C$, layer IV. $N_{v}$ is the number of neurons per $1 \mathrm{~mm}^{3}$ of tissue (in thousands) 


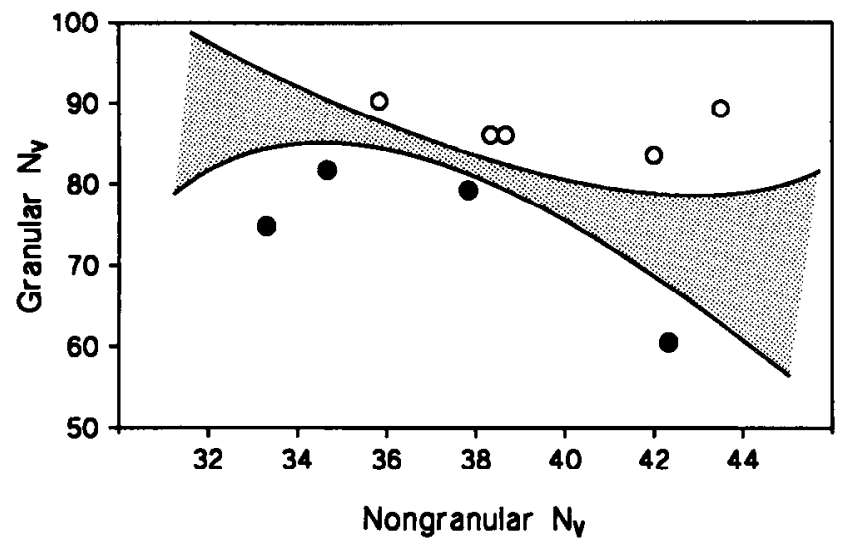

Figure 7. Scatterplot of $N_{\mathrm{r}}$ for granular versus nongranular systems for each female (open circles) and each male (solid circles) brain. The granular values are the means of layers II and IV, the nongranular values are the means of layers III, V, and VI. Scores are means of values for the right and left hemispheres. The stippled area highlights the separation between the sexes. There is no overlap between the sexes for the granular system, in contrast to the almost complete overlap for the nongranular system. $N_{i}$ is the number of neurons per $1 \mathrm{~mm}^{3}$ of tissue (in thousands).

has generally been argued to have little biological significance on the basis of no sex differences in general cognitive abilities such as overall intelligence or memory (Halpern, 1992) and similar ratios of brain size to measures of body size in men and women (Gould, 1981). However, the assumption of an allometric relationship is undermined by the very low correlations between brain and body size variables within male and fenale groups (Peters, 1991), and also by the different pattern of sex differences observed for different cortical layers (discussed in a subsequent section). Cortical metabolic rate (expressed per unit volume) has been found to be greater in women than men in some PET studies (e.g., Hatazawa et al., 1987). Our results suggest that the greater $N_{v}$ in female cortex may contribute to this metabolic difference.

\section{Granular versus nongranular systems}

The laminar analyses revealed a disparity in the pattern of similarities and differences between the sexes for layers II and IV (the granular system) and layers III, V, and VI (the nongranular system). Our small sample size likely provided insufficient power to detect all the laminar differences that may exist. Differences of at least $10 \%$ which did not reach statistical significance but which might be of biolugical importance are discussed as tendencies. Figure 8 presents a summary of the sex differences in the two systems. This set of findings requires a multifactorial model of the origin of sex differences in cortical microscopic structure. In the granular system, $N$, was greater in women (by $17 \%)$, resulting from similar cortical depth and a tendency (15\%) for greater $N_{c}$ compared to men. The disparity in $N_{v}$ between women and men suggests different connectivity in granular layers. The combination of the tendency for area $\mathrm{T} A_{1}$ to be greater in men $(17 \%)$ and their lower $N_{v}$ resulted in a similar estimated total number of neurons in layers II and IV in men and women. The pattern of sex differences in the granular system fits with the compression model.

In the nongranular system, there was no evidence of reliable sex differences in cortical depth, $N_{\mathrm{c}}$ or $N_{v}$. The larger area TA in men combined with a similar $N_{v}$ to women resulted in a ten-
Table 3. Means ${ }^{x}$ and SDs for total number of neurons $\left(N_{T}\right)$ in the estimated cortical volume of area $\mathrm{T} \boldsymbol{A}_{1}$ for total cortex and for each layer for each sex

\begin{tabular}{|c|c|c|c|c|c|}
\hline \multirow[b]{2}{*}{ Layers } & \multicolumn{2}{|c|}{$N_{1}$ (in millions) } & \multirow[b]{2}{*}{$\begin{array}{l}F-M \% \\
\text { Differencer }\end{array}$} & \multicolumn{2}{|c|}{$\begin{array}{l}\text { Each layer as \% of } \\
\text { total } N_{1}\end{array}$} \\
\hline & $\begin{array}{l}\text { Female } \\
\bar{X}(\mathrm{SD}) \\
\end{array}$ & $\begin{array}{l}\text { Male } \\
\bar{X}(\text { SD) }\end{array}$ & & $\begin{array}{l}\text { Female } \\
\bar{X}\end{array}$ & $\begin{array}{l}\text { Male } \\
\bar{X}\end{array}$ \\
\hline I & $3(1)$ & $4(2)$ & -29.6 & 2.5 & 3.0 \\
\hline II & $23(8)$ & $25(13)$ & -8.3 & 19.2 & 18.5 \\
\hline III & $36(16)$ & $44(16)$ & -20.0 & 30.0 & 32.6 \\
\hline IV & $24(7)$ & $23(9)$ & 4.3 & 20.0 & 17.0 \\
\hline V & $18(8)$ & $21(8)$ & -15.4 & 15.0 & 15.6 \\
\hline VI & $16(7)$ & $18(6)$ & -11.8 & 13.3 & 13.3 \\
\hline Total & $120(44)$ & $135(46)$ & -10.9 & 100.0 & 100.0 \\
\hline
\end{tabular}

"Scores are the means of the right and left hemisphere values.

"For all comparisons, sample size was five women and four men; for each $t$ test, df $=7$ and $p>0.35$.

c $[(F-M) /[(F+M) / 2]] \times 100$.

dency for the estimated total number of neurons to be greater in men $(17 \%)$. This pattern suggests that factors other than those operative in the granular system are in effect here. The difference in the cytoarchitectonic characteristics of area $\mathrm{T} A_{1}$ between men and women is highlighted by the different correlations between granular $N_{v}$ and nongranular $N_{v}$ in men $(r=-0.78)$ and in women ( $r=-0.29$ ) (see Fig. 7, Results).

Interestingly, in a study of sex differences in cell packing density in the rat, Reid and Juraska (1992) showed that the total number of neurons was greater in the male than female brain in binocular primary visual cortex in all layers except layer IV. Although the authors emphasized the sex differences that were found, of particular relevance to the present result is that only in layer IV, the rat's granular layer, was depth not greater in males and $\operatorname{did} N_{v}$ tend to be larger in females, resulting in similar total neuron number. The general point is that although these studies deal with very different species and cytoarchitectonic areas, sex differences in neuronal density may be specific to granular layers across mammalian species.

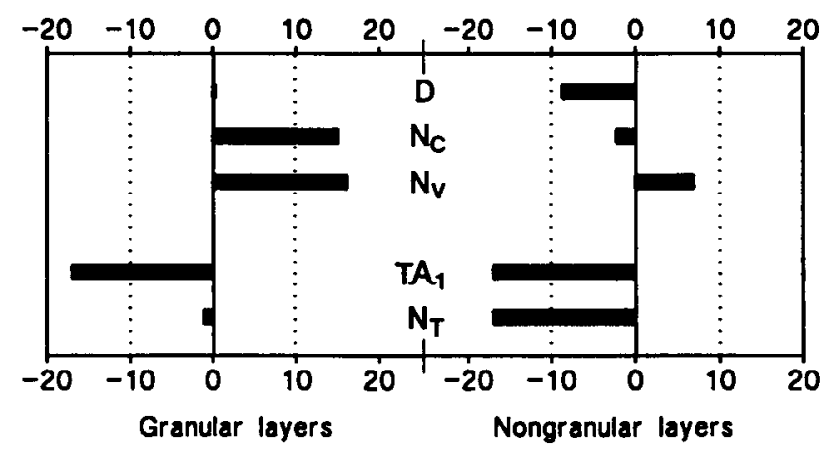

\section{F-M \% Difference}

Figure 8 . Summary chart showing percent difference between male $(M)$ and female $(F)$ brains in cortical depth $(D), N_{c}, N_{1}$, estimated total surface area of region $\mathrm{T} A_{1}$ and estimated total number of neurons $\left(N_{T}\right)$ in the cortical volume of area $T A_{1}$ for the granular (layers II and IV) and nongranular systems (layers III, V, and VI). Scores are means of right and left hemispheres. The percent difference score is $[(F-M) /$ $[(F+M) / 2]] \times 100$. The dotted lines highlight differences of at least $10 \%$. 


\section{Differential sexual differentiation of the cortical layers}

The presence of sex differences in the microscopic structure of $\mathrm{T} A$, cortex suggests that some sex-related genetic or epigenetic factors play a role in their development. For $\mathrm{T} A_{1}$ cortex, as in the case of other organs which undergo sexual differentiation, female cortex may be prototypic (i.e., determined predominantly by genetic factors) whereas male cortex would be a derivative resulting from the operation of additional biologic factors, such as sex steroids. The presence of different patterns of sex differences and similarities in the laminar systems (granular vs nongranular) suggests that sexual differentiation of the cortex must be different in some respect for the different layers. In any hypothetical model, some factor may result in the greater total number of nongranular cells (by greater proliferation or less cell death) in men than women which, in combination with the greater area of the cytoarchitectonic region in the male brain, results in similar $N_{v}$ values. In the granular system the process must work differently, resulting, possibly by default, in similar total numbers of cells which, when packed into the smaller region in the female brain, leads to the greater $N_{v}$ in women.

Tracing and immunocytochemical studics indicate that layers II and IV compared to layers III, V, and VI have neuronal populations which have different functions in terms of input and output and which synthesize different transmitters (Naegele and Barnstable, 1989). The granular system is predominantly an input component of the cortical column and contains mostly GABAergic neurons. The nongranular system is mainly an output system with most neurons being glutamate positive. Our results suggest that the cortical functional unit is constituted differently in each sex and that at least for auditory association cortex, men and women who are right-handed differ in the histologic nature of the input layers and in the proportional amounts of the input versus output systems or of the GABAergic versus glutamate systems. This suggests greater thalamocortical flow in women than men. It is not difficult to imagine that such differences could influence receptive fields and have consequences for specific psychologic skills such as aspects of stimulus discrimination or perceptual integration. Delineation of the neuroanatomic substrate of cognition may be furthered by studies which examine the possible cognitive correlates of histologic variables which show individual differences.

\section{Expanded cytoarchitectonic map of area TA,}

Cytoarchitectonic area $\mathrm{T} A$, was reliably identified in each hemisphere, in either the horizontal, vertical, or both walls of the posterior region of the superior temporal gyrus in the Sylvian fissure (see Fig. 1). Our finding that the same cytoarchitectonic region is present in the vertical as in the horizontal surface of the posterior Sylvian fissure supports the conclusion based on gross anatomy that the planum temporale includes the vertical segment in the posterior ascending ramus of the Sylvian fissure (e.g., Rubens, 1976; Witelson and Kigar, 1992). This vertically oriented cortex has not been mapped cytoarchitectonically before. Von Economo and Horn (1930) analyzed only the horizontal supratemporal cortex. Blinkov (1949) and Galaburda et al. (1978) used coronal sections which often are oblique or even parallel to the vertical wall. In the latter report, study of the coronal sketches suggests that $\mathrm{T} A$, type tissue (referred to as $\mathrm{Tpt}$ tissue) was sometimes present in cortex on the lateral aspect of the brain adjacent to the vertical upswing of the Sylvian fissure, which buttresses our finding. Definition of the planum temporale including both horizontal and vertical components revealed right-left symmetry in overall size of this region (Witelson and Kigar, 1991, 1992; Loftus et al., 1993), in contrast to the numerous observations of a greater planum temporale on the left side when only the horizontal plane was considered. Clarification and definition of the gross anatomy of this region is important, since currently various definitions of the gross anatomy of this gyral surface are being used in the increasing number of brain imaging studies relating anatomical and psychological measures in normal (e.g., Steinmetz et al., 1991) and clinical groups (e.g., Leonard et al., 1993).

\section{References}

$\Lambda$ boitiz F, Scheibel AB, Zaidel E (1992) Morphometry of the Sylvian fissure and the corpus callosum, with emphasis on sex differences. Brain 115:1521-1541.

Allen LS, Gorski RA (1991) Sexual dinorphism of the anterior commissure and massa intermedia of the human brain. J Comp Neurol 312:97-104.

Allen LS, Richey MF, Chai YM, Gorski RA (1991) Sex differences in the corpus callosum of the living human being. J Neurosci 11:933942.

Annett M (1967) The binomial distribution of right, mixed and left handedness. Q J Exp Psychol 19:327-333.

Blinkov SM (1949) The temporal region. In: Cytoarchitectonics of the human cerebral cortex (in Russian) (Sarkisov SA, Filimonov IN, eds), pp 344-380. Moscow: Medgiz.

Blinkov SM, Glezer II (1968) The human brain in figures and tables. New York: Plenum.

Braendgaard H, Evans SM, Howard CV, Gundersen HJG (1990) The total number of neurons in the human cortex unbiasedly estimated using optical disectors. J Microsc 157:285-304.

Brodmann K (1909) Vergleichende Lokalisationslehre der Großhirnrinde in ihren Prinzipien dargestellt auf Grund des Zellenbaues. Leipzig: Barth.

Coggeshall RE (1992) A consideration of neural counting methods. Trends Neurosci 15:9-13.

Dekaban AS, Sadowsky D (1978) Changes in brain weights during the span of human life: relation of brain weights to body heights and body weights. Ann Neurol 4:345-356.

Denenberg VH, Kertesz A, Cowell PE (1991) A factor analysis of the human's corpus callosum. Brain Res 548:126-132.

Galaburda A, Sanides F (1980) Cytoarchitectonic organization of the human auditory cortex. J Comp Neurol 190:597-610.

Galaburda AM, Sanides F, Geschwind N (1978) Human brain. Cytoarchitectonic left-right asymmetries in the temporal speech region. Arch Neurol 35:812-817.

Geschwind N, Levitsky W (1968) Human brain: left-right asymmetries in temporal speech region. Science 161:186-187.

Gould SJ (1981) The mismeasure of man, Chap 3. New York: Norton.

Habib M, Gayraud D, Oliva A, Regis J, Salamon G, Khalil R (1991) Effects of handedness and sex on the morphology of the corpus calIosum: a study with brain magnetic resonance imaging. Brain Cognit $16: 41-61$.

Halpern DF (1992) Sex differences in cognitive abilities, Chap 3, 2d ed. Hillsdale, NJ: Erlbaum.

Hatazawa J, Brooks RA, Di Chiro G, Campbell G (1987) Global cerebral glucose utilization is independent of brain size: a PET study. J Cump Assist Tomugr 11:571-576.

Hécaen H, Albert ML (1978) Human neuropsychology. New York: Wiley.

Kimura D) (1987) Are men's and women's brains really different? Can Psychol 28:133-147.

Leonard CM, Voeller KKS, Lombardino LJ, Morris MK, Hynd GW, Alexander AW, Andersen HG, Garofalakis M, Honeyman JC, Mao J, Agee OF, Staab EV (1993) Anomalous cerebral structure in dyslexia revealed with magnetic resonance imaging. Arch Neurol 50:461-469.

Loftus WC, Tramo MJ, Thomas CE, Green RL, Nordgren RA, Gazzaniga MS (1993) Three-dimensional quantitative analysis of hemispheric asymmetry in the human superior temporal region. Cereb Cortex 3:348-355.

McEwen BS (1983) Gonadal steroid influences on brain development 
and sexual differentiation. In: Reproductive physiology IV, Vol 27 (Greep R, ed), pp 99-145. Baltimore: University Park.

Naegele JR, Barnstable CJ (1989) Molecular determinants of GABAergic local-circuit neurons in the visual cortex. Trends Neurosci $12: 28-34$

Pakkenberg B (1993) Total nerve cell number in neocortex in chronic schizophrenics and controls estimated using optical disectors. Biol Psychiatry 34:768-772.

Pakkenberg B, Evans SM, Møller A, Brændgaard H, Jørgen H, Gundersen $G$ (1989) Total number of neurons in human neocortex related to age and sex estimated by way of optical disectors. Acta Stereol 8:251-256.

Pandya DN, Seltzer B (1986) The topography of commissural fibers. In: Neurology and neurobiology, Vol 17, Two hemispheres-one brain (Leporé F, Ptito M, Jasper HH, eds), pp 47-73. New York: Liss.

Peters M (1991) Sex differences in human brain size and the general meaning of differences in brain size. Can J Psychol 45:507-522.

Rakic P (1988) Specification of cerebral cortical areas. Science 241: 170-176.

Reid SNM, Juraska JM (1992) Sex differences in neuron number in the binocular area of the rat visual cortex. J Comp Neurol 321:448 455.

Rockel AJ, Hiorns RW, Powell TPS (1980) The basic uniformity in structure of the neocortex. Brain 103:221-244.

Rubens AB, Mahowald MW, Hutton JT (1976) Asymmetry of the lateral (sylvian) fissures in man. Neurology 26:620-624.

Steinmetz H, Volkmann J, Jäncke L, Freund H-J (1991) Anatomical left-right asymmetry of language-related temporal cortex is different in left- and right-handers. Ann Neurol 29:315-319.

Steinmetz H, Jäncke L, Kleinschmidt A, Schlaug G, Volkmann J, Huang $Y$ (1992) Sex but no hand difference in the isthmus of the corpus callosum. Neurology 42:749-752.

Terry RD, DeTeresa R, Hansen LA (1987) Neocortical cell counts in normal human adult aging. Ann Neurol 21:530-539.

von Economo C, Horn L (1930) Über Windungsrelief, Maße und Rin- denarchitektonik der Supratemporalfläche, Ihre individuellen und Ihre Seitenunterschiede. Z Gesamte Neurol Psychiatr 130:678-757.

von Economo C, Koskinas GN (1925) Die Cytoarchitektonik der Hirnrinde des erwachsenen Menschen. Berlin: Springer.

Wada JA, Clarke R, Hamm A (1975) Cerebral hemispheric asymmetry in humans. Cortical speech zones in 100 adult and 100 infant brains. Arch Neurol 32:239-246.

Weschler D (1955) Manual: Weschler adult intelligence scale. New York: Psychological Corporation.

Witelson SF (1987) Brain asymnetry, functional aspects. In: Encyclopedia of neuroscience (Adelman G, ed), pp 152-156. Cambridge, MA: Birkhauser.

Witelson SF (1989) Hand and sex differences in the isthmus and genu of the human corpus callosum: a postmortem morphological study. Brain 112:799-835.

Witelson SF, Goldsmith CH (1991) The relationship of hand preference to anatomy of the corpus callosum in men. Brain Res 545:175-182.

Witelson SF, Kigar DL (1988) Asymmetry in brain function follows asymmetry in anatomical form: gross, microscopic, postmortem and imaging studies. In: Handbook of neuropsychology, Vol 1 (Boller F, Grafman J, eds), pp 111-142. Amsterdam: Elsevier

Witelson SF, Kigar DL (1991) Anatomy of the planum temporale in relation to side, handedness and sex. Soc Neurosci Abstr 17:414.3.

Witelson SF. Kigar DL (1992) Sylvian fissure morphology and asymmetry in men and women: bilateral differences in relation to handedness in men. J Comp Neurol 323:326-340.

Witelson SF, Kigar DL (1993) Anterior commissure in relation to corpus callosum anatomy, hand preference and sex. Soc Neurosci Abstr 19:232.6.

Witelson SF, McCulloch PB (1991) Premortem and postmortem measurement to study structure with function: a human brain collection. Schizophr Bull 17:583-591.

Witelson SF, Pallie W (1973) Left hemisphere specialization for language in the newborn: anatomical evidence of asymmetry. Brain 96: $641-646$.

Witelson SF, Kigar DL, McKanna JA (1992) A computer-assisted direct-imaging system to obtain numerical densities of neurons in human cortex. Br Res Bull 29:441-447. 